\title{
Heading Control of an AUV Based on Mamdani Fuzzy Inference
}

\author{
Zaopeng Dong*, Lei Wan, Tao Liu \& Jiayuan Zhuang
}

Science and Technology on Autonomous Underwater Vehicle, Harbin Engineering University, Harbin 150001, China

*Corresponding author: dongzaopeng@hrbeu.edu.cn

Keywords: autonomous underwater vehicle (AUV), heading control, fuzzy control technology, Mamdani Fuzzy Inference(MFI), Gaussian membership function.

\begin{abstract}
A class of autonomous underwater vehicle (AUV) heading control problem is addressed in this paper based on Mamdani Fuzzy Inference (MFI). Firstly, empirical knowledge of heading control for ship from experienced captain is fuzzed into fuzzy control rules, which can be identified by computer, using fuzzy set theory. Secondly, output of the controller is derived by calculating the excitation of system input to the control rules, combing with the application of multi-input-multi-rule MFI. Thirdly, robust adaptive heading controller for AUV is designed incorporating the distributing characteristics of Gaussian membership function, in order to overcome randomness and uncertainty of the external environment disturbances on AUV. At last, simulation experiment is carried out to verify the effectiveness and superiority of the controller designed.
\end{abstract}

\section{Introduction}

Autonomous underwater vehicles (AUVs) have a wide range of applications in marine geosicence, and are increasingly being used in the scientific, military, commercial, and policy sectors [1-3]. Their ability to operate autonomously of a host vessel makes them well suited to extreme environments, from the world's deepest hydrothermal vents to beneath polar ice sheets. They have revolutionized our ability to image the seafloor, providing higher resolution seafloor mapping data than can be achieved from surface vessels, particularly in deep water [4-6]. Heading control is one of the most important motion control problems of AUV, it will directly affect the stability and reliability of the AUV operations, especially in target tracking, obstacle avoiding. Many researchers from all over the world have paid their attention to heading control problem of AUV in few decades.

A sliding mode control algorithm for heading control of an AUV is presented in [7] using hydrodynamic coefficients, which are estimated by using traditional nonlinear sliding mode observer and extender Kalman filter. In paper [8], an integrated RS-Chaos-LSSVM model is proposed in order to overcome uncertainties of the environment and impreciseness of model, which is used in the nonlinear and time-varying heading control system. Yan Zheping and his team designed an extension integral variable structure heading controller for AUV system in[9], which combing the idea of intelligent control technology with traditional integral variable structure control technology by introducing extension logic into the algorithm.

In order to deal with the disturbance of ocean currents and pulse moment in the heading control system of AUV, Xu Jian designed an auto-disturbance-rejection heading controller in [10], where a linear heading control model is obtained by linearization. A $\mathrm{H} \infty$ controller based on state observers is designed to solve the unobservable problem of partial condition in heading control system of AUV by using the linear matrix inequality processing method in[11], while a hybrid controller, which combines the good qualities of both neural network controller and PID controller in order to make maximum profit from both techniques, is proposed in [12] to deal with AUV heading control problem.

Fuzzy control technology is a kind of technology which does not rely on the mathematical model of controlled object, is widely used in our daily life such as automatic washing machine, metro control, and many other industrial process control problems. Thus fuzzy controller is very suitable 
for heading control of an AUV as it is hard for us to establish precise mathematical model. Moreover as Gaussian membership function with the characteristic of normal distribution, we combined Gaussian function with fuzzy control technology and then proposed a simple and effective MFI based controller for heading control of an AUV.

The paper is organized as follows. In Section 1, we introduce the some backgrounds of AUV research and show some heading controller for an AUV designed before by some counterparts, Section2 describes the first-order nonlinear heading response model of an AUV. Section 3 is devoted to design MFI based controller. Simulations of the AUV heading controller designed in Section 3 is taken in Section 4. At last, some concluding remarks of the paper are made in Section 5.

\section{Nonlinear model of an AUV}

The following first-order nonlinear equation of yawing response describes the nonlinear heading control system of an AUV, as the steering is not very frequent. See Equation 1 below [13]:

$$
\left\{\begin{array}{l}
\dot{\varphi}=r \\
\dot{r}=-\frac{1}{T} r-\frac{\alpha}{T} r^{3}+\frac{K}{T} \delta+w
\end{array}\right.
$$

Where $\varphi$ is heading angle, $r$ is yaw angular velocity, $T$ is stability constant, $K$ is turning constant, $\alpha$ is nonlinear coefficient of the model, $\delta$ is rudder angle, $w$ represents uncertainties of the system.

\section{Controller design}

Heading control system of AUV can be described as follows, see in Figure 1. The control input of the Mamdani fuzzy controller are deviation of heading angle and its differential while the control output is rudder angle. Mamdani fuzzy controller design process mainly consists of four parts, namely fuzzification, control rules, fuzzy inference and defuzzification, they are detail designed in section 3.1 to section 3.4 respectively.

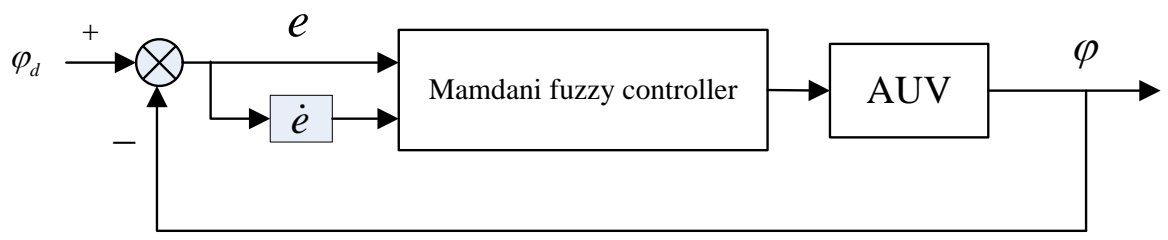

Figure 1. Heading control system of AUV

Fuzzification. Gaussian membership function is one of the most important membership functions for fuzzification, because of its distribution characteristics, it enhances the ability of resisting the external environment and other uncertainty interferences for the control system. Let $x^{*}$ be a known precise variable, $\tilde{A}^{*}$ is a fuzzy set fuzzed with the variable ${ }^{x}$, and then the mathematical expression of Gaussian membership function is:

$$
\mu_{\tilde{A}^{*}}(x)=e^{-\frac{\left(x-x^{*}\right)^{2}}{2 \sigma^{2}}}
$$

Where $\sigma$ is a positive parameter, which determines the steepness of the Gaussian function. Control input of Mamdani fuzzy control which are deviation of heading angle and its differential, and control output which is rudder angle can all be fuzzed as follows using Gaussian membership function, see Figure 2, where the range of the variables is normalized to a range of $[-6,6]$, corresponding fuzzy variables sets are [NB, NM, NS, ZO, PS, PM, PB]. 


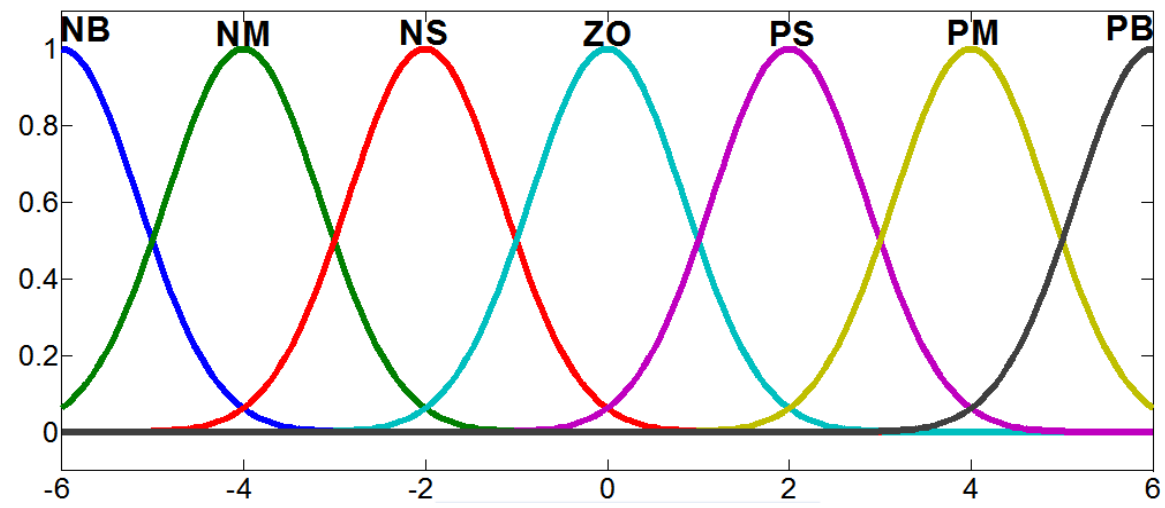

Figure 2. Fuzzification of variables

Fuzzy control rules. Fuzzy control rules is the most important part of the fuzzy controller, it will directly affect the performance of the controller. According to the previous setting of fuzzy sets for variables, we convert the experience of manipulating rudder by experienced captain to fuzzy control rules which can be recognized by fuzzy controller, see table 1 . In table 1 , field 1 is deviation of heading angle, field 2 presents differential of heading angle deviation while field 3 means rudder angle of AUV.

Table 1. Fuzzy control rules

\begin{tabular}{c|c|c|c|c|c|c|c}
\hline 3 & NB & NM & NS & ZO & PS & PM & PB \\
\hline NB & NB & NB & NM & NB & NM & PS & PB \\
\hline NM & NB & NB & NM & NB & NS & PS & PB \\
\hline NS & NB & NB & NS & NM & ZO & PM & PB \\
\hline ZO & NB & NB & NS & ZO & PS & PM & PB \\
\hline PS & NB & NB & ZO & PM & PM & PB & PB \\
\hline PM & NB & NM & PS & PB & PM & PB & PB \\
\hline PB & NB & NM & PS & PB & PB & PB & PB \\
\hline
\end{tabular}

Mamdani Fuzzy Inference (MFI). Mamdani Fuzzy Inference (MFI) is the most widely used fuzzy inference in fuzzy controller design, its inference process with multi-rules can be shown as follows, take two rules inference for example:

Table 2 Mamdani Fuzzy Inference (MFI)

Rule 1 If $x$ is $\tilde{A}_{1}$ and $y$ is $\tilde{B}_{1}$ then $z$ is $\tilde{C}_{1}$

Rule 2 If $x$ is $\tilde{A}_{2}$ and $y$ is $\tilde{B}_{2}$ then $z$ is $\tilde{C}_{2}$

Premise $\quad x$ is $\tilde{A}^{*}$ and $y$ is $\tilde{B}^{*}$

Conclusion $z$ is $\tilde{C}^{*}$

Let $\tilde{A}^{*}, \tilde{A}_{1}, \tilde{A}_{2}, \widetilde{B}^{*}, \widetilde{B}_{1}, \widetilde{B}_{2}$ and $\widetilde{C}^{*}, \widetilde{C}_{1}, \widetilde{C}_{2}$ be fuzzy sets for $X, Y$ and $Z$ which to be discussed, respectively. $\quad \tilde{R}_{M 1}(X, Y, Z)$ represents the fuzzy relationship among $\tilde{A}_{1}, \tilde{B}_{1}$ and $\tilde{C}_{1}$, while $\tilde{R}_{M 2}(X, Y, Z)$ represents that among $\tilde{A}_{2}, \widetilde{B}_{2}$ and $\tilde{C}_{2}$. MFI would inference for $\tilde{C}^{*}$ in $Z$ from $\tilde{A}^{*}$ and $\tilde{B}^{*}$ which are known in $X$ and $Y$, combing with control rules design before. MFI can be shown as follows, see table 2 .

For multi-input-multi-rule fuzzy inference problem, we usually deal it with the union of the fuzzy relationship for each single fuzzy rule, then fuzzy arithmetic with the unions. Thus MFI show in table 2 can be expressed as follows: 


$$
\begin{aligned}
& \mu_{\tilde{C}^{*}}(z)=\underset{\substack{x \in \mathrm{X} \\
y \in \mathrm{Y}}}{\mathrm{V}}\left[\mu_{\tilde{A}^{*}}(x) \Lambda \mu_{\tilde{B}^{*}}(y)\right] \Lambda\left[\mu_{\tilde{R}_{\tilde{M}_{1}}}(x, y, z) \mathrm{V} \mu_{\tilde{R}_{\tilde{R}^{2}}}(x, y, z)\right]
\end{aligned}
$$

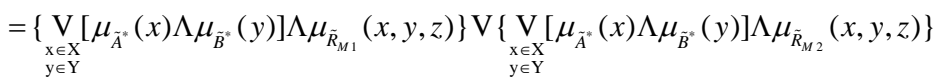

$$
\begin{aligned}
& =\mu_{\tilde{C}_{1}^{*}}(z) \mathrm{V} \mu_{\tilde{C}_{L^{*}}^{*}}(z)
\end{aligned}
$$

Where $\mu_{\tilde{R}_{M 1}}(x, y, z)=\mu_{\tilde{A}_{1}}(x) \Lambda \mu_{\tilde{R}_{1}}(x) \mathrm{V} \mu_{\tilde{C}_{1}}(z), \mu_{\tilde{R}_{M 2}}(x, y, z)=\mu_{\tilde{A}_{2}}(x) \Lambda \mu_{\tilde{B}_{2}}(x) \mathrm{V} \mu_{\tilde{C}_{2}}(z), \mu_{\tilde{C}_{1}}(z)$ represents the union under the control of fuzzy rule $1, \mu_{\tilde{C}_{2}^{*}}(z)$ represents the union under the control of fuzzy rule 2. They can simply be described as follows, see figure 3:

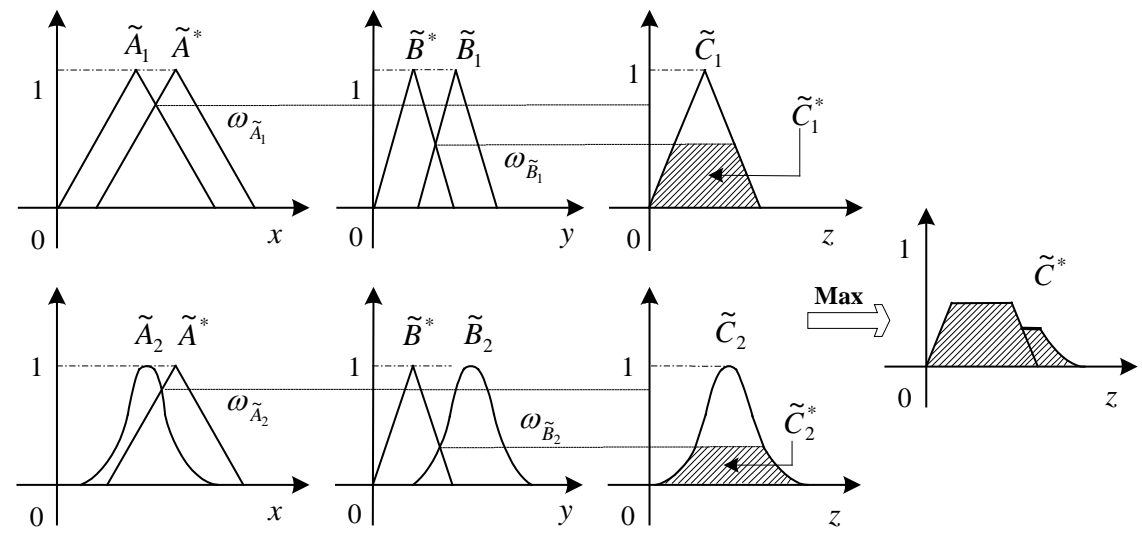

Figure 3: Mamdani fuzzy inference process

Defuzzification. Defuzzificaion for AUV heading control here is chosen as area centroid method, usually referred to centroid method, the most outstanding feature of it is that it combines all of the results from fuzzy inference, so the clarity value that obtain could improve the controller with better stability and anti-jamming performance. Centroid method for defuzzification can be expressed as follows:

$$
y^{*}=\frac{\int_{y_{\min }}^{y_{\max }} y \mu(y) d y}{\int_{y_{\min }}^{y_{\max }} \mu(y) d y}
$$

Where $y^{*}$ represents the coordinate value of the center of overall area, namely clarity value that we need, $\mu(y)$ represents the membership function of $y$ in $Y$.

\section{Simulation experiment}

In order to demonstrate the effectiveness and stability of the MFI based fuzzy controller designed in this paper, we take an AUV model whose parameters are shown in table 3 for heading control simulation experiment. We have $0.15 \sin (2 \pi \mathrm{t})$ expressed the external sea condition influence on AUV, while $1 / T$ represented the model uncertainties and other random disturbances, see table 3:

Table 3. Parameters of USV model

\begin{tabular}{|c|c|c|c|}
\hline parameter & value & parameter & value \\
\hline$K$ & -2.36 & $\alpha$ & 0.000094 \\
\hline$T$ & 5.489 & $w$ & $\pm \frac{1}{T} \mathrm{deg} / \mathrm{s}^{2}+0.15 \sin (0.2 \pi t)$ \\
\hline
\end{tabular}

The initial heading angle of AUV is set as 0, while the desired heading angle is set as 90deg and 120deg, respectively in case 1 and case 2. Heading control experiments on AUV are simulated under the controller designed by traditional PID control technology and Mamdani fuzzy control technology proposed in this paper for comparison, results from two methods are shown below in figure 4 and figure 5 for case 1 and case 2:

We can see from the results show in figure 4 and figure 5 that PID controller could not well control heading of AUV in the presence of complex sea condition, random disturbances and model 
uncertainties, while MFI based fuzzy controller designed in this paper shows well control performance in heading control of AUV, with higher accuracy, better stability and stronger reliability.

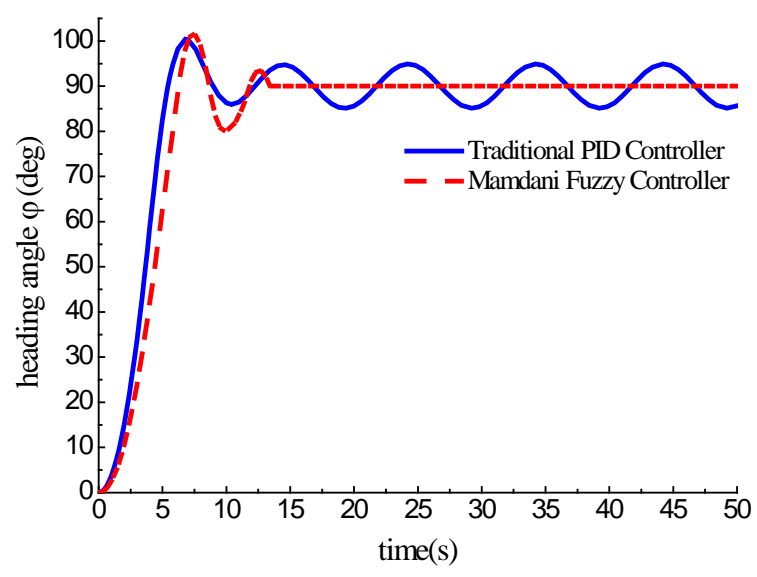

Figure 4 Heading control results in case 1

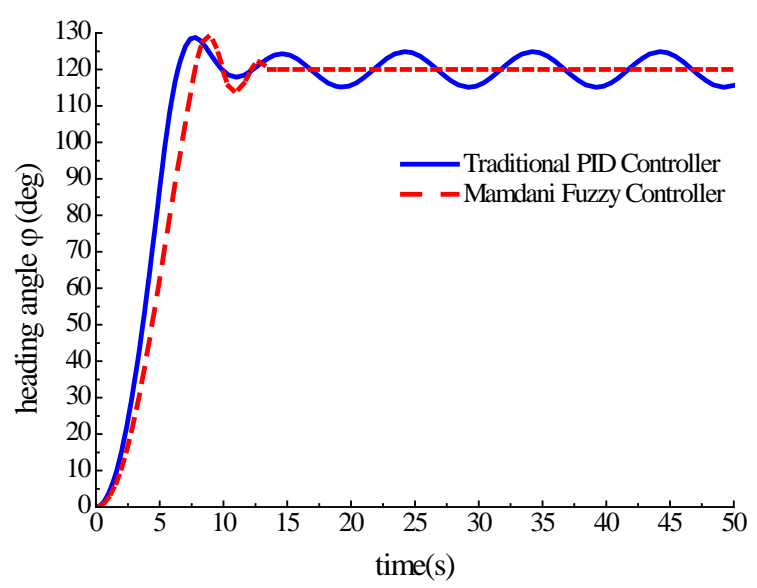

Figure 5 Heading control results in case 2

In addition, it is easy to see that overshoot and oscillations appear in the heading control of AUV with Mamdani fuzzy controller, but eventually stabilized. That is mainly because of the design of the fuzzy control rules for Mamdani fuzzy controller are not very fine, which can only give a rough estimate. At the same time the fuzzy set domain division of the variables is not detail enough, increasing of the number of fuzzy set domain divisions would improve the performance of the controller and reduce overshoot and oscillations while that would lead to complexity of controller rules and difficulty of MFI. Considering the trade-offs between the performance and the complexity of the controller, we design the controller above and acceptable overshoot and oscillations appear.

\section{Conclusions}

A MFI based fuzzy controller is designed for heading control of AUV considering the external sea condition influence, model uncertainties and other random disturbances in this paper. Comparing with the traditional PID control technology, Mamdani fuzzy control technology which is mainly derived from human experience shows better control performance in heading control of AUV, with higher accuracy, better stability and stronger reliability. Moreover acceptable overshoot and oscillations appear because of the considering of reducing the complexity and difficulty controller design process, which expected to be solved in the next project combing the neural network control technology. 


\section{Acknowledgements}

Thanks for the support of National Natural Science Foundation of China under Grant No. 51409054 and 51409059 and National High Technology Research and Development Program 863 of China under Grant No. 2012AA09A304

\section{References}

[1] Li Ye, Jiang Yan-Qing \& Ma Shan. Inverse speed analysis and low speed control of underwater vehicle. Journal of Central South University. 21(7), pp. 2652-2659, 2014

[2] Alam K., Ray T. \& Anavatti S.G. Design and construction of an autonomous underwater vehicle. Neurocomputing, 142(SI), pp.16-29, 2014

[3] Loc M.B, Choi H.S \& Seo J.M. Development and control of a new AUV platform. International Journal of Control Automation and Systems, 12(4), pp.886-894, 2014.

[4] Zhang Tie-Dong, Wan Lei \& Zeng Wen-Jing. Object dection and tracking method of AUV based on acoustic vision. China Ocean Engineering, 26(4), pp.623-636, 2012.

[5] Villar S.A, Acosta G.G \& Sousa A.L. Evaluation of an efficient approach for target tracking from acoustic imagery for the perception system of an autonomous underwater vehicle. International Journal of Advanced Robotic Systems, 11, pp.1-13, 2014

[6] Wynn R. B, Huvenne V. A. I \& Le Bas T. P. Autonomous underwater vehicles(AUVs): Their past, present and future contributions to the advancement of marine geoscience. Marine Geology, 352, pp.451-468, 2014.

[7] Kim J, Kim K \& Choi H. S. Depth and heading control for autonomous underwater vehicle using estimated hydrodynamic coefficients. Oceans Conference Record(IEEE), 2001, pp.429-435.

[8] Song Xiao-Ru, Xue Yong-Gang \& Luo De-Zhu. Analysis on AUV heading control system based on RS-Chaos-LSSVM. Sensors and Transducers. 155(8), pp.162-170, 2013.

[9] Yan Zhe-Ping, Wu Di, Zhou Jia-Jia \& Zhang Xun. Application of extension integral variable structure control method on simulation of AUV heading control system. Proceedings of the 30th Chinese Control Conference, 2011, pp.2617-2621.

[10] Xu Jian, Yan Zhe-Ping \& Zhao Jie. The AUV heading control system based on auto disturbance rejection control principle. 2010 IEEE International Conference on Information and Automation, 2010, pp.1018-1022.

[11] Luo Yue-Sheng, Wen Xiu-Ping \& Li Shi-Wei. H $\infty$ heading control of AUV based on state observers. Proceedings of the 2nd International Conference on Future Control and Automation, 1, pp.165-174, 2012.

[12] Xia Guo-Qing, Li Tang \& Guo Feng-Shui. Design of a hybrid controller for heading control of an autonomous underwater vehicle. Proceedings of the IEEE International Conference on Industrial Technology, 2009

[13] Fossen T.I. Handbook of marine craft hydrodynamics and motion control, United Kingdom: John Wiley \& Sons, 2011. 